\title{
Global prevalence and epidemiology of Strongyloides stercoralis in dogs: a systematic review and meta-analysis
}

\author{
Aida Vafae Eslahi ${ }^{1 \dagger}$, Sima Hashemipour ${ }^{2 \dagger}$, Meysam Olfatifar ${ }^{1 \dagger}$, Elham Houshmand ${ }^{3}$, Elham Hajialilo ${ }^{4,5}$, \\ Razzagh Mahmoudi ${ }^{1}$, Milad Badri ${ }^{*}$ and Jennifer K. Ketzis ${ }^{6^{*}}$ (I)
}

\begin{abstract}
Background: Strongyloides stercoralis, a soil-transmitted helminth, occurs in humans, non-human primates, dogs, cats and wild canids. The zoonotic potential between these hosts is not well understood with data available on prevalence primarily focused on humans. To increase knowledge on prevalence, this review and meta-analysis was performed to estimate the global status of S. stercoralis infections in dogs.
\end{abstract}

Methods: Following the PRISMA guidelines, online literature published prior to November 2020 was obtained from multiple databases (Science Direct, Web of Science, PubMed, Scopus and Google Scholar). Prevalence was calculated on a global and country level, by country income and climate, and in stray/animal shelter dogs versus owned dogs. Statistical analyses were conducted using R-software (version 3.6.1).

Results: From 9428 articles, 61 met the inclusion criteria. The estimated pooled global prevalence of S. stercoralis in dogs was 6\% (95\% Cl 3-9\%). Infection was found to be the most prevalent in low-income countries with pooled prevalence of $22 \%(95 \% \mathrm{Cl} 10-36 \%)$. The highest pooled prevalence of S. stercoralis in dogs was related to regions with average temperature of $10-20{ }^{\circ} \mathrm{C}(6 \% ; 95 \% \mathrm{Cl} 3-11 \%)$, an annual rainfall of $1001-1500 \mathrm{~mm}(9 \% ; 95 \% \mathrm{Cl} 4-15 \%)$ and humidity of $40-75 \%$ (8\%; 95\% Cl 4-13\%). Prevalence was higher in stray and shelter dogs (11\%; 95\% Cl 1-26\%) than in owned dogs (3\%; 95\% Cl 1-7\%).

Conclusions: As with S. stercoralis in humans, higher prevalence in dogs is found in subtropical and tropical regions and lower-income countries, locations which also can have high dog populations. While this study presents the first estimated global prevalence of S. stercoralis in dogs, it is potentially an underestimation with 15 of 61 studies relying on diagnostic methods of lower sensitivity and a paucity of data from most locations. Standardized protocols (e.g. quantity of feces and number of samples for a Baermann) in future studies could improve reliability of results. More prevalence studies and raising veterinary awareness of $S$. stercoralis are needed for a One Health approach to protect humans and dogs from the impact of the infection.

Keywords: Strongyloides stercoralis, Canine, Neglected tropical disease, Soil transmitted helminth, Systematic review

*Correspondence: Badri22.milad@gmail.com; JKetzis@rossu.edu

${ }^{\dagger}$ Aida Vafae Eslahi, Sima Hashemipour and Meysam Olfatifar contributed equally to this work

${ }^{1}$ Medical Microbiology Research Center, Qazvin University of Medical Sciences, Qazvin, Iran

${ }^{6}$ Ross University School of Veterinary Medicine, Basseterre, West Indies, St. Kitts and Nevis

Full list of author information is available at the end of the article

\section{Background}

A quarter of the world's population is impacted by helminthic infections that cause substantial rates of diseases and/or disabilities. Many of these helminths are zoonotic with carnivores, particularly dogs and cats, responsible for transmission of nearly $43 \%$ of the zoonotic pathogens original author(s) and the source, provide a link to the Creative Commons licence, and indicate if changes were made. The images or other third party material in this article are included in the article's Creative Commons licence, unless indicated otherwise in a credit line to the material. If material is not included in the article's Creative Commons licence and your intended use is not permitted by statutory regulation or exceeds the permitted use, you will need to obtain permission directly from the copyright holder. To view a copy of this licence, visit http://creativecommons.org/licenses/by/4.0/. The Creative Commons Public Domain Dedication waiver (http://creativeco mmons.org/publicdomain/zero/1.0/) applies to the data made available in this article, unless otherwise stated in a credit line to the data. 
[1-4]. One of these zoonotic pathogens is Strongyloides stercoralis, a soil-transmitted helminth that affects 100370 million people globally and is classified as a neglected tropical disease [5, 6]. The main manifestations of S. stercoralis infection are gastrointestinal and cutaneous signs. However, S. stercoralis infections can be asymptomatic but, in the other extreme, can cause severe pulmonary pathology with auto- and hyperinfection [7-9]. The life cycle of $S$. stercoralis involves homogonic and heterogonic stages. In the homogonic cycle, only females exist in the host and eggs are produced via parthenogenesis. First-stage larvae (L1) and occasionally in some hosts eggs containing L1 are excreted via the host's feces into the environment where the heterogonic cycle occurs. Male and female larvae molt into free-living adult worms through four larval stages with female larvae also being able to molt into infectious third-stage larvae (L3) with no further development until entering a host [8]. The most common techniques to detect larvae of S. stercoralis in human feces are direct smear, Kato-Katz, flotation, sedimentation, Baermann and Koga agar plate culture with the latter three being the more sensitive for larvae. Indirect fluorescent antibody tests (IFATs), enzymelinked immunosorbent assays (ELISAs) and molecular methods also can be used for diagnosis but are more frequently used in research versus clinical settings [1012]. The primary treatment for $S$. stercoralis infection in humans is ivermectin [9].

Strongyloides stercoralis also is capable of infecting canids and a range of other vertebrate hosts such as felids and non-human primates $[6,13]$. Canine S. stercoralis infection occurs most frequently in puppies and young dogs under 1 year of age and in puppies living in breeding kennels with poor sanitary conditions during hot and humid seasons [14-18]. Infection in dogs can be asymptomatic, but also can be life-threatening with clinical signs ranging from diarrhea and malabsorption to bronchopneumonia. Extraintestinal disseminations such as the nasal cavities, lungs, stomach and cranial cavity associated with severe clinical signs have been documented in immunocompromised canids as in humans (e.g. due to other pre-existing conditions or administration of immunosuppressive medicines) [19-21]. The methods for detection of S. stercoralis in dog feces are the same as those in humans with treatments including not only ivermectin but also fenbendazole, albendazole and selamectin although no products are registered for this use in dogs [22].

Dogs and humans share certain S. stercoralis genotypes. Although there are few reports of transmission from dogs to humans, experimental infections illustrate that S. stercoralis from human origin can infect dogs, suggesting dogs can be a reservoir for human infection
$[6,13,23,24]$. While there have been recent studies estimating regional and global prevalence of Strongyloides for humans and associated risk factors [5, 7, 12, 25], these data are not available for infections in dogs. In a one health context, better knowledge on the prevalence of S. stercoralis infection in dogs and the risk of zoonotic transmission to humans is needed. Therefore, this review and meta-analysis aimed to estimate the global prevalence of S. stercoralis in dogs and assess some variables that might influence prevalence.

\section{Methods \\ Search strategy}

This systematic review and meta-analysis followed PRISMA guidelines (http://www.prisma-statement.org/). A systematic literature search was carried out on multiple general science databases to identify all publications reporting $S$. stercoralis in dogs across the world published prior to November 2020. Science Direct, Web of Science, PubMed, Scopus and Google Scholar were explored using the following search terms: Strongyloides stercoralis, S. stercoralis, strongyloidiasis, dogs, puppies, gastrointestinal helminths, soil-transmitted helminths, worldwide and prevalence using AND and/or OR Boolean operators. Two independent authors involved in the search evaluated titles and abstracts and reviewed the full-text papers. After removing duplicates and irrelevant records, reference lists of full texts were examined for potential eligibility of citations not found in the database search.

\section{Inclusion and exclusion criteria and data extracted}

Literature was eligible for inclusion if it met the following priori criteria: (1) peer-reviewed articles containing original data, (2) cross-sectional studies reporting the prevalence of strongyloidiasis in dogs, (3) accessible full text and abstract and (4) numerator and denominator data available to confirm prevalence values. Literature that did not satisfy the aforementioned criteria, such as review articles with no original data, letters, editorials, articles with fecal material collected from the ground and lack of clarity about whether there were repeated samples and articles with ambiguous/undetermined conclusions, were excluded. Articles that reported S. stercoralis in humans, animals other than dogs and soil were excluded.

Using a Microsoft Excel ${ }^{\circledR}$ spreadsheet, the following information was retrieved from the included articles: first author name, year of publication, country where the study was conducted, continent, sample size and number of positive cases, diagnostic method(s) used, income level (https://datahelpdesk.worldbank.org/knowledgeb ase/articles/906519-world-bank-country-and-lendinggroups), humidity (https://www.timeanddate.com/weath er/iran/tehran/climate), annual rainfall (https://en.clima 
te-data.org/), average temperature (https://en.climatedata.org/), latitude, (https://www.geodatos.net/en/coord inates/) and climate (https://www.britannica.com/scien ce/Koppen-climate-classification). Antibody seroprevalence studies were excluded, since they cannot confirm current infection. Experimental antigen methods also were excluded. In studies where more than one method was used to analyze a single sample (e.g. Baermann and flotation), the total number of positive samples was determined and used in the analysis.

\section{Quality assessment}

The Newcastle-Ottawa Scale was used to assess the quality of the included articles [26]. A maximum score of 9 was assigned to each article based on subject selection (0-4 points), comparability of subjects $(0-2$ points) and exposure (0-3 points). A total score of $0-3,4-6$ and 7-9 points was considered poor, moderate and high quality, respectively $[27-29]$.

\section{Data synthesis and statistical analysis}

The pooled prevalence of S. stercoralis in dogs reported globally and by continent was calculated with $95 \%$ confidence intervals (CIs). In addition, prevalence for stray and shelter dogs was compared to that of owned dogs with owned dogs defined as household owned, those in pet stores and breeding dogs but excluding those with only breeding kennel data. Sub-group analysis included country income level, humidity, annual rainfall, average temperature and latitude. The probability of publication bias was surveyed using Egger's regression test and Begg's test. A meta-regression analysis was conducted to evaluate the impact of the year of publication on prevalence. All statistical analyses were performed using the meta-package in $R$ (version 3.6.1). The pooled prevalence estimates were computed using the alpha method for the random-effects model, based on the inverse variance approach for measuring weight. Cochrane's $Q$ test and inconsistency index ( $I^{2}$ statistics) were used to assess the magnitude of heterogeneity among included studies, with $I^{2}$ values of $<25 \%, 25-75 \%$ and $<75 \%$ considered as low, moderate and high heterogeneity, respectively. A $P$ value $<0.05$ was considered statistically significant.

\section{Results}

\section{Literature search, selection and data extraction}

Our systematic search yielded a total of 9428 publications. One hundred thirty-two full-text articles were chosen for eligibility assessment. There were 16 studies with an unspecified sample size and 55 studies with no original data, including reviews, case reports and case series, letters, theses and workshops. Finally, 61 studies were included in the meta-analysis based on critical appraisal criteria (Fig. 1; Table 1 with full list of references in Additional file 1: References S1). The included studies utilized parasitology techniques comprising microscopic methods (flotation with and without concentration, sedimentation, Baermann, Kato-Katz and other direct smear methods, and necropsy), culture methods (Harada-Mori and agar plate culture), molecular techniques [conventional polymerase chain reactions (PCR) and real-time PCR] and serological methods (IFAT and ELISA). Of the 61 articles, 15 used only direct smears and/or flotation with most studies using sedimentation and/or Baermann often in combination with flotation or direct smears. Four studies used culture, three of which were combined with other methods. Only eight of the included articles used diagnostic methods other than microscopy and culture: three used immunological methods in addition to microscopic or culture methods; one used serology, PCR, microscopic and culture methods; one used PCR and microscopic methods; and one used only PCR methods.

\section{Pooled prevalence}

The estimated pooled global prevalence for S. stercoralis in dogs was 6\% (95\% CI 3-9\%) with a higher estimated pooled prevalence in stray/shelter dogs $(11 \%$, $95 \%$ CI 1-26\%) than in owned dogs (3\%, 95\% CI 1-7\%) (Figs. 2; 3). Based on the manuscripts included in the analysis, S. stercoralis infection in dogs has been documented in 29 countries (Fig. 4; Additional file 2: Figure S1). The pooled prevalence on different continents ranged from 21 to $2 \%$, with $21 \%$ (95\% CI $10-34 \%)$ in Africa, 6\% (95\% CI 0-100\%) in Oceania, 5\% (95\% CI 0-14\%) in Asia, 2\% (95\% CI 0-10\%) in North America, $2 \%(95 \%$ CI $0-7 \%)$ in South America and 2\% (95\% CI 0-5\%) in Europe (Fig. 2; Additional file 3: Table S1).

The largest number of studies was conducted in Ethiopia (8 studies), followed by Japan (6 studies). Analyses based on countries showed that Cambodia had the highest pooled prevalence (85\%, 95\% CI 78-91\%) (Additional file 2: Figure S1). The estimated pooled prevalence based on country-level income groups ranged from 22 to $2 \%$, with the highest rate in lowincome countries $(22 \%, 95 \%$ CI $10-36 \%)$ (Additional file 3: Table S1).

Our analyses revealed that regions with average temperatures of $10-20{ }^{\circ} \mathrm{C}$ had the highest prevalence of S. stercoralis $(6 \%, 95 \%$ CI 3-11\%) (Additional file 3: Table S1). Furthermore, the infection was more prevalent in regions with humidity of $40-75 \%(8 \%, 95 \%$ CI $4-13 \%)$, annual rainfall of $1001-1500 \mathrm{~mm}(9 \%, 95 \%$ CI 4-15\%) and a tropical wet and dry climate (12\%, 95\% CI 5-21\%). In addition, we found that the highest prevalence rate was at a latitude of $1^{\circ}-25^{\circ}(11 \%, 95 \%$ CI 5-19\%). 


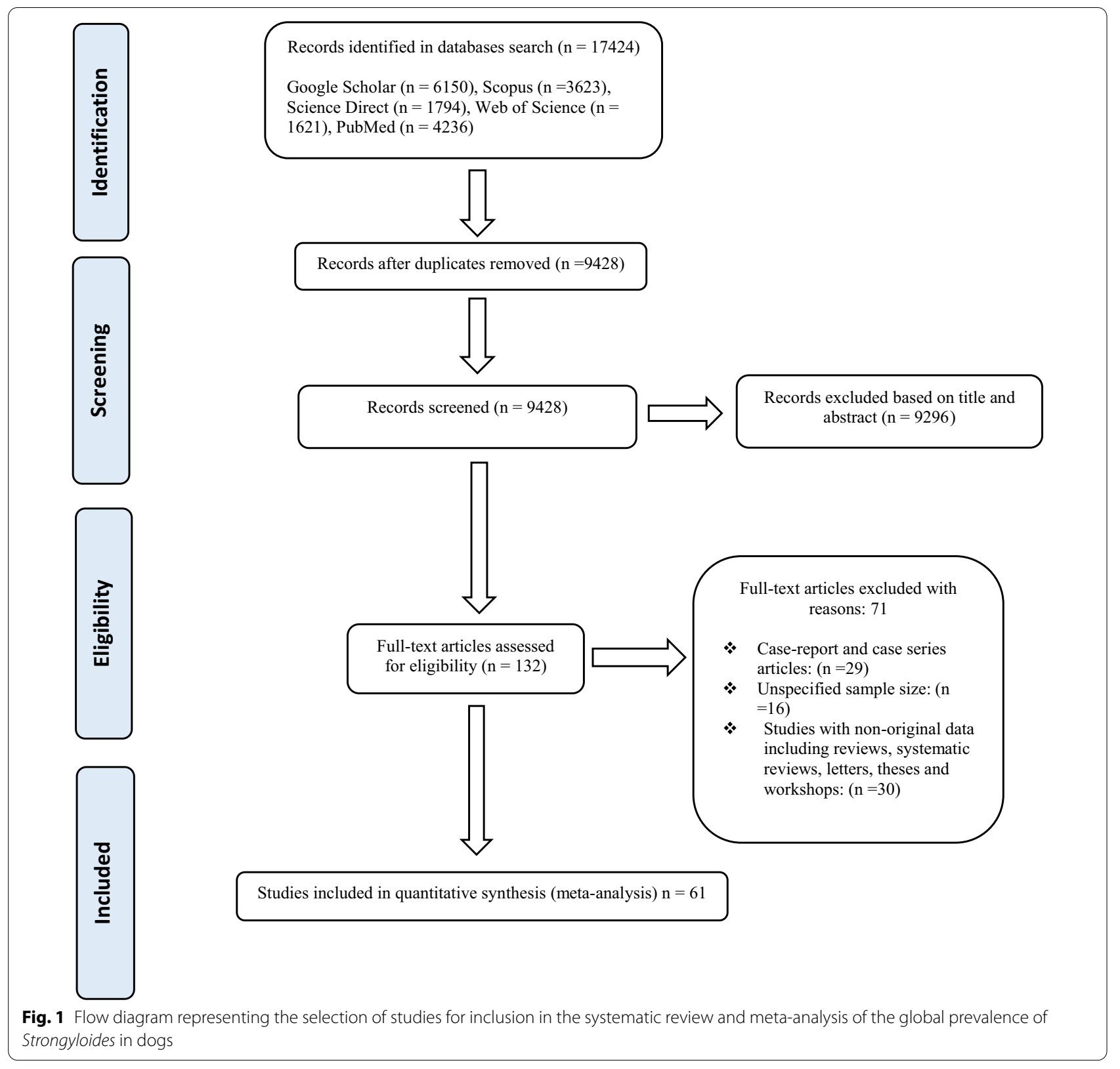

\section{Publication bias}

As demonstrated by funnel plot asymmetry, a highly significant publication bias was observed in our study using Egger's test $(t=4.12, P=0.0001)$ and Begg's test $(P=0.001)$ (Fig. 5A, B). Meta-regression analysis demonstrated that there was a significant heterogeneity between studies regarding the year of publication (regression slope $=0.0055, P=0.0116$ ) (Fig. 6). Evaluation of study quality revealed that, among 61 studies, 36 had a total score of
7-9 points (high quality) and 25 had a total score of 4-6 points (moderate quality). No included studies were considered poor quality (Table 1).

\section{Discussion}

Dogs are among the most popular companion animals with a considerable positive impact on the psychological and physiological conditions of their owners [30, 31]. This close relationship with dogs, however, can pose 
Table 1 Main characteristics of the included studies reporting the prevalence of Strongyloides stercoralis in dogs

\begin{tabular}{|c|c|c|c|c|c|c|}
\hline No. & First author & Publication year & Country & Continent & Diagnostic methods used & $\begin{array}{l}\text { Quality assessment based } \\
\text { on Newcastle-Ottawa } \\
\text { Scale }\end{array}$ \\
\hline 1 & Rizzo and Ricciardi & 1978 & Italy & Europe & Unclear & 5 \\
\hline 2 & Ugochukwu and Ejimadu & 1985 & Nigeria & Africa & $\begin{array}{l}\text { Saturated solution flotation, formalin- } \\
\text { ether sedimentation }\end{array}$ & 5 \\
\hline 3 & Tarish et al. & 1986 & Iraq & Asia & Necropsy & 6 \\
\hline 4 & Stehr-Green et al. & 1987 & USA & North America & Formalin-ethyl acetate sedimentation & 9 \\
\hline 5 & Epe et al. & 1993 & Germany & Europe & Coproscopical examinations & 6 \\
\hline 6 & Bugg et al. & 1999 & Australia & Oceania & $\begin{array}{l}\text { Sedimentation, zinc sulfate }\left(\mathrm{ZnSO}_{4}\right) \\
\text { flotation }\end{array}$ & 7 \\
\hline 7 & Itoh et al. & 2003 & Japan & Asia & Coproscopical examination & 7 \\
\hline 8 & Anosike et al. & 2004 & Nigeria & Africa & Direct smear, concentration methods & 5 \\
\hline 9 & Asano et al. & 2004 & Japan & Asia & $\begin{array}{l}\text { Direct smear, saturated salt ( } \mathrm{NaCl} \text { ) flota- } \\
\text { tion, } \mathrm{ZnSO}_{4} \text { flotation, sucrose flotation }\end{array}$ & 8 \\
\hline 10 & Ramirez-Barrios et al. & 2004 & Venezuela & South America & $\mathrm{NaCl}$ flotation & 8 \\
\hline 11 & Komtangi et al. & 2005 & Cameroon & Africa & McMaster & 5 \\
\hline 12 & Júnior et al. & 2006 & Brazil & South America & Baermann, sedimentation, ELISA, IFAT & 9 \\
\hline 13 & Gonçalves et al. & 2007 & Brazil & South America & Baermann, sedimentation, ELISA, IFAT & 8 \\
\hline 14 & Lorenzini et al. & 2007 & Brazil & South America & Saturated $\mathrm{NaCl}$ flotation, $\mathrm{ZnSO}_{4}$ flotation & 6 \\
\hline 15 & Papazahariadou et al. & 2007 & Greece & Europe & Teleman's sedimentation & 8 \\
\hline 16 & Dillard et al. & 2007 & Finland & Europe & Baermann & 7 \\
\hline 17 & Ugbomoiko et al. & 2008 & Nigeria & Africa & Kato-Katz thick smear & 7 \\
\hline 18 & Das et al. & 2009 & India & Asia & Unclear & 5 \\
\hline 19 & Claerebout et al. & 2009 & Belgium & Europe & Sucrose flotation & 8 \\
\hline 20 & Gates and Nolan & 2009 & USA & North America & $\begin{array}{l}\mathrm{ZnSO}_{4} \text { flotation, formalin-ethyl acetate } \\
\text { sedimentation }\end{array}$ & 8 \\
\hline 21 & Itoh et al. & 2009 & Japan & Asia & Formalin-ethyl acetate sedimentation & 7 \\
\hline 22 & Takano et al. & 2009 & Japan & Asia & Direct smear, agar plate culture (APC) & 7 \\
\hline 23 & Leelayoova et al. & 2009 & Thailand & Asia & $\begin{array}{l}\text { Direct smear, formalin-ethyl acetate } \\
\text { concentration }\end{array}$ & 6 \\
\hline 24 & Razmi & 2009 & Iran & Asia & Mini Parasep ${ }^{\circledR}$ SF (sedimentation) & 7 \\
\hline 25 & Mariana et al. & 2010 & Bolivia & South America & Willis-Malloy flotation & 5 \\
\hline 26 & Zewdu et al. & 2010 & Ethiopia & Africa & Necropsy & 6 \\
\hline 27 & Awoke et al. & 2011 & Ethiopia & Africa & Direct smear, flotation & 5 \\
\hline 28 & Jones et al. & 2011 & Ethiopia & Africa & Necropsy & 5 \\
\hline 29 & Itoh et al. & 2011 & Japan & Asia & Formalin-ethyl acetate sedimentation & 8 \\
\hline 30 & Itoh et al. & 2011 & Japan & Asia & Formalin-ethyl acetate sedimentation & 7 \\
\hline 31 & Paulos et al. & 2012 & Ethiopia & Africa & Sedimentation and flotation & 5 \\
\hline 32 & Martins et al. & 2012 & Brazil & South America & PARATEST ${ }^{\circledR}$ Diagnostek (sedimentation) & 7 \\
\hline 33 & Getahun and Addis & 2012 & Ethiopia & Africa & Sedimentation and $\mathrm{NaCl}$ flotation & 6 \\
\hline 34 & Mircean et al. & 2012 & Romania & Europe & $\mathrm{NaCl}$ flotation & 8 \\
\hline 35 & Mekbib et al. & 2013 & Ethiopia & Africa & Direct smear, flotation and sedimentation & 5 \\
\hline 36 & G/selasie et al. & 2013 & Ethiopia & Africa & McMaster, sedimentation & 5 \\
\hline 37 & Abere et al. & 2013 & Ethiopia & Africa & $\begin{array}{l}\text { Direct smear, sedimentation and } \mathrm{NaCl} \\
\text { flotation }\end{array}$ & 6 \\
\hline 38 & Perera et al. & 2013 & Sri Lanka & Asia & $\begin{array}{l}\mathrm{NaCl} \text { flotation, Sheather's sucrose flota- } \\
\text { tion, direct smear }\end{array}$ & 6 \\
\hline 39 & Riggio et al. & 2013 & Italy & Europe & Flotation, Baermann & 8 \\
\hline 40 & Ortuno et al. & 2014 & Spain & Europe & $\mathrm{ZnSO}_{4}$ flotation & 7 \\
\hline 41 & Alvarado-Esquivel et al. & 2015 & Mexico & North America & Sheather's and $\mathrm{ZnSO}_{4}$ flotation & 8 \\
\hline
\end{tabular}


Table 1 (continued)

\begin{tabular}{|c|c|c|c|c|c|c|}
\hline No. & First author & Publication year & Country & Continent & Diagnostic methods used & $\begin{array}{l}\text { Quality assessment based } \\
\text { on Newcastle-Ottawa } \\
\text { Scale }\end{array}$ \\
\hline 42 & Puebla et al. & 2015 & Cuba & North America & $\begin{array}{l}\text { Direct smear, formalin ethyl acetate sedi- } \\
\text { mentation, Kato-Katz smear, Willy-Malloy } \\
\text { flotation }\end{array}$ & 5 \\
\hline 43 & Elom et al. & 2015 & Nigeria & Africa & $\begin{array}{l}\mathrm{NaCl} \text { and } \mathrm{ZnSO}_{4} \text { floatation Formol ether } \\
\text { sedimentation }\end{array}$ & 5 \\
\hline 44 & Hadi and Faraj & 2016 & Iraq & Asia & $\begin{array}{l}\text { Direct smear, potassium dichromate } \\
\mathrm{K}_{2} \mathrm{Cr}_{2} \mathrm{O}_{4} \text { film, } \mathrm{NaCl} \text { flotation, Formalin- } \\
\text { ether sedimentation }\end{array}$ & 5 \\
\hline 45 & Wright et al. & 2016 & UK & Europe & FLOTAC technique & 7 \\
\hline 46 & Ferreira et al. & 2016 & Brazil & South America & $\begin{array}{l}\text { Sucrose and } \mathrm{NaCl} \text { flotation, water-ether } \\
\text { sedimentation }\end{array}$ & 7 \\
\hline 47 & Pumidonming et al. & 2016 & Thailand & Asia & $\begin{array}{l}\text { Flotation, formalin-ethyl acetate sedimen- } \\
\text { tation }\end{array}$ & 7 \\
\hline 48 & Strkolcova et al. & 2017 & Slovakia & Europe & Flotation, Baermann, APC, ELISA & 7 \\
\hline 49 & Paradies et al. & 2017 & Italy & Europe & Direct smear, Baermann, necropsy & 8 \\
\hline 50 & Mircean et al. & 2017 & Romania & Europe & $\mathrm{NaCl}$ flotation, sedimentation & 8 \\
\hline 51 & Jaleta et al. & 2017 & Cambodia & Asia & Baermann, Kato-Katz & 9 \\
\hline 52 & Sauda et al. & 2018 & Italy & Europe & Flotation, Baermann & 8 \\
\hline 53 & García et al. & 2018 & Venezuela & South America & $\mathrm{NaCl}$ flotation & 5 \\
\hline 54 & Hurtado and Forero & 2019 & Colombia & Asia & Formalin-gasoline concentration & 5 \\
\hline 55 & latta et al. & 2019 & Italy & Europe & Direct smear, Baermann, APC, IFAT, RT-PCR & 8 \\
\hline 56 & Sanchez-Thevenet et al. & 2019 & Spain & Europe & $\begin{array}{l}\text { Modified Ritchie formalin-ether, Sheath- } \\
\text { er's sugar flotation, RT-PCR }\end{array}$ & 8 \\
\hline 57 & Kurnosova et al. & 2019 & Russia & Europe & $\mathrm{NaCl}$ and ammonium nitrate flotations & 7 \\
\hline 58 & Sanpool et al. & 2020 & Thailand & Asia & APC & 7 \\
\hline 59 & Beknazarova et al. & 2020 & Australia & Oceania & qPCR, RT-PCR & 8 \\
\hline 60 & Dashchenko et al. & 2020 & Ukraine & Europe & $\begin{array}{l}\text { Direct smear, Baermann, modified string } \\
\text { test }\end{array}$ & 6 \\
\hline 61 & Nagamori et al. & 2020 & USA & North America & $\begin{array}{l}\text { Direct smear, flotation, sedimentation, } \\
\text { Baermann }\end{array}$ & 8 \\
\hline
\end{tabular}

certain risks with the potential of dogs transmitting a broad range of zoonotic pathogens, including viruses, bacteria, parasites and fungi [32-34]. Regarding S. stercoralis, some genotypes are dog specific while others can infect dogs and humans; hence, dogs could be reservoirs of zoonotic S. stercoralis $[6,13,35]$. In this review and meta-analysis, we estimated the global prevalence of $S$. stercoralis in dogs. Our findings show $S$. stercoralis in dogs being documented in 29 countries and that infections are not limited to dogs in tropical and subtropical regions, although these regions have the higher prevalence as is the general case for human infections [12].

Factors that potentially contribute to the higher prevalence in tropical regions include temperature and humidity, country income and presence of stray dogs. Similar to prior studies, the results of the meta-analysis herein presented indicate that climate conditions play a key role in the prevalence of $S$. stercoralis infection with the highest pooled prevalence in areas with a tropical wet and dry climate [12]. The higher humidity and temperature make favorable conditions for survival of heterogonic stages of $S$. stercoralis [36, 37]. While the climate plays a key role in prevalence, several studies have confirmed higher rates of helminthic infections in humans in low-income countries, attributed to sanitary conditions and limited access to health care [38-40]. This is similar to our results, with the highest prevalence of S. stercoralis in dogs in low-income countries. In these countries, 
Study

Africa

Komtangi et al 2005 Cameroo

Zewdu et al 2010 Ethiopia

Awoke et al 2011 Ethiopia

Jones et al 2011 Ethiopia

Paulos et al 2012 Ethiopia

Getahun and Addis 2012 Ethiopia

Mekbib et al 2013 Ethiopia

selasie et al 2013 Ethiopia

Abere et al 2013 Ethiopia

Elom et al 2015 Nigeria

Ugochukwu and Ejimadu 1985 Nigeria

Anosike et al 2004 Nigeria

Ugbomoiko et al 2008 Nigeria

Random effects model

Heterogeneity: $I^{2}=98.9866 \%, \tau^{2}=0.0570, p<.001$

Asia

Jaleta et al 2017 Cambodia

Das et al 2009 India

Razmi 2009 Iran

Hadi and Faraj 2016 Iraq

Tarish et al 1986 Iraq

Itoh et al. 2003 Japan

Asano et al 2004 Japan

Itoh et al 2011 Japan

Itoh et al 2011 Japan

Takano et al 2009 Japa

Takano et al $2009 \mathrm{Jap}$

Itoh et al 2009 Japan

Perera ct a 2013 Sri Lanka

Pumidoning et al 2016 Thailan

Sanpool ct al 2020 Thail land

Leelayoova et at 2009 Thailand

Random effects mode

Heterogeneity: $I^{2}=97.742 \%, \tau^{2}=0.0729, p<.00$

Europe

Claerebout et al 2009 Belgium

Dillard et al 2007 Finland

Epe et al 1993 Germany

Papazahariadou et al 2007 Greece

Riggio et al 2013 Italy

Paradies et al 2017 Italy

Sauda et al 2018 Italy

Iatta et al 2019 Italy

Rizzo and Ricciardi 1978 Italy

Mircean et al 2012 Romanis

Mircean et al 2017 Romani

Kurnosova et al 2019 Russi

Strkolcova et al 2017 Slovakia

Sanchez-Thevenet et al 2019 Spai

Ortuno et al 2014 Spain

Wright et al $2016 \mathrm{UK}$

Dashchenko et al 2020 Ukraine

Random effects model

Heterogeneity: $I^{2}=93.5863 \%, \tau^{2}=0.0195, p<.001$

North America

Puebla et al 2015 Cuba

Alvarado-Esquivel et al 2015 Mexico

Stehr-Green et al 1987 USA

Gates and Nolan 2009 USA

Nagamori et al 2020 USA

Nagamori et al 2020 USA

Heterogeneity: $I^{2}=93.3025 \%, \tau^{2}=0.0205, p<.001$

Oceania

Bugg et al 1999 Australi

Beknazarova et al 2020 Austral

Random effects model

Heterogencity: $I^{2}=99.2156 \%, \tau^{2}=0.0945, p<001$

South America

Mariana et al 2010 Bolivia

Martins et al 2012 Braril

Meris

J'init 12006 B

Jinior 2006 B

Gonçalves et al 2007 Brazi

Lorenzini et al 2007 Brazil

Hurtado and Forero 2019 Colo

Garcia et al 2018 Venezuela

Ramirez-Barrios et al $2004 \mathrm{~V}$
Random effects model

Heterogeneity: $I^{2}=96.4645 \%, \tau^{2}=0.0237, p<.001$

Random effects model

Heterogeneity: $I^{2}=99.0421 \%, \tau^{2}=0.0558, p<.001$

Test for subgroup differences: $\chi_{5}^{2}=20.7336, \mathrm{df}=5(p<.001)$
Prevalence

$95 \% \mathrm{CI}$

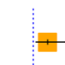

$+$

$+$

7
$+\square$
$+\quad 0.4610$
$+\quad 0.5002$

$[0.1852 ; 0.3380]$

$[0.0258 ; 0.0708]$

$[0.4024 ; 0.5207]$

$[0.1779 ; 0.2600]$

$[0.5681 ; 0.6334]$

$[0.2649 ; 0.3500]$

[0.1994: 0.2796]

$[0.0103 ; 0.0845]$

$[0.1110 ; 0.1987]$

$[0.0835,0.1349]$

$0.1065 \quad[0.0835 ; 0.1349]$

$\mathbf{0 . 2 1 5 4} \quad[0.1093 ; 0.3453]$

$+$

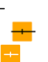

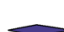

$0.8547 \quad[0.7796 ; 0.9073]$

$0.0429 \quad[0.0210 ; 0.0860]$

$0.0287 \quad[0.0123 ; 0.0655]$

$0.0500 \quad[0.0231 ; 0.1048]$

$0.0500 \quad[0.0089 ; 0.2361]$

$0.0186 \quad[0.0129 ; 0.0268]$

$0.0104 \quad[0.0053 ; 0.0203]$

$0.0111 \quad[0.0072 ; 0.0172]$

$[0.0012 ; 0.0055]$

[0.0395; 0.1958$]$

$[0.0395 ; 0.1958]$

$[0.0062 ; 0.0189]$

$[0.0615 ; 0.1926]$

$[0.0052 ; 0.0438]$

$[0.0179 ; 0.0938]$

$[0.0070 ; 0.1426$

$0.0009 \quad[0.0002 ; 0.0049]$

$0.0652 \quad[0.0224 ; 0.1750]$

$0.0030 \quad[0.0016 ; 0.0055]$

$0.0178 \quad[0.0076 ; 0.0410]$

$0.0084 \quad[0.0023 ; 0.0300]$

$0.0221 \quad[0.0101 ; 0.0473]$

$[0.0003 ; 0.0088]$

$0.3600-\quad[0.2727 ; 0.4576]$

$0.0059-[0.0010,0.0326]$

$0.0385-[0.0106,0.1298]$

$\quad 0.0350-\quad 0.0263 ; 0.0464]$

$0.0091 \quad[0.0056 ; 0.0148]$

$0.1200 \quad[0.0562 ; 0.2380]$

$0.0114 \quad[0.0039 ; 0.0330]$

$0.0118 \quad[0.0033 ; 0.0421]$

$0.0175 \quad[0.0060 ; 0.0503]$

$0.0275 \quad[0.0150 ; 0.0498]$

$0.0255[0.0074 ; 0.0541]$

$0.0206 \quad[0.0057 ; 0.0721]$

$0.1584 \quad[0.0999 ; 0.2419]$

$0.0140 \quad[0.0038 ; 0.0496]$

$0.0020 \quad[0.0012: 0.0034]$

$0.0020 \quad[0.0012 ; 0.0033]$

$0.0215[0.0000 ; 0.1061]$

$0.0024 \quad[0.0004 ; 0.0133]$

$0.2190 \quad[0.1741 ; 0.2716]$

$0.0699[0.0000 ; 1.0000]$

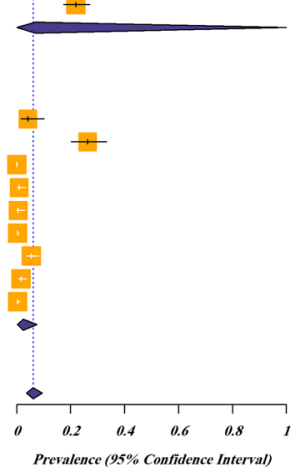

$0.0417 \quad[0.0163 ; 0.1023]$

$0.2632 \quad[0.2029 ; 0.3338]$

$0.0010 \quad[0.0003 ; 0.0028]$

0.0093 [0.0026; 0.0333$]$

$\quad 0.0055 \quad[0.0010 ; 0.0306]$

0.0041 [0.0019; 0.0089]

$0.0546 \quad[0.0367 ; 0.0806]$

$0.0163 \quad[0.0070 ; 0.0377]$

$\begin{array}{cc}0.0033 & {[0.0009 ; 0.0118]} \\ \mathbf{0 . 0 2 4 5} & {[\mathbf{0 . 0 0 1 3} ; \mathbf{0 . 0 7 5 3}}\end{array}$

$0.0618 \quad[0.0356 ; 0.0945]$

Fig. 2 Forest plots for random-effects meta-analysis of the global prevalence of Strongyloides stercoralis in dogs based on continent 


\section{Owned dogs}

Rizzo and Ricciardi. 1978 Italy

Stchr-Green et al. 1987 USA

Bugg ct al. 1999 Australia

Itoh et al. 2003 Japan

Anosike et al. 2004 Nigeria

Asano et al. 2004 Japan

Ramirez-Barrios et al. 2004 Venezuela

Komtangi et al. 2005 Cameroon

Júnior et al. 2006 Braril

Gonçalves et al. 2007 Brazil

Papazahariadou et al. 2007 Greece

Dillard et al. 2007 Finland

Ugbomoiko et al. 2008 Nigeria

Claerebout et al. 2009 Belgium

Gates and Nolan. 2009 USA

Itoh et al. 2009 Japan

Razmi et al. 2009 Iran

Takano ct al. 2009 Japan

Leclayoova ct al. 2009 Thailand

Mariana ct al. 2010 Bolivia

Itoh et al. 2011 Japan

Itoh et al. 2011 Japan

Getahun and Addis. 2012 Ethiopia

Martins et al. 2012 Brayil

Riggio et al. 2013 Italy

Ortuno et al. 2014 Spain

Elom et al. 2015 Nigeria

Wright et al. 2016 UK

Pumidonming et al. 2016 Thailand

Ferreira et al. 2016 Brazil

Jaleta et al. 2017 Cambodia

Kurnosova et al. 2019 Russia

Hurtado and Forcro. 2019 Colombia

Sanpool et al. 2020 Thailand

Nagamori ct al. 2020 USA

Random effects model

Heterogeneity: $I^{2}=97.7682 \%, \tau^{2}=0.0454, p<.001$

Stray / animal shelter dogs

Ng and Kelly. 1975 Australia

Tarish et al. 1986 Iraq

Das et al. 2009 India

Zewdu et al. 2010 Ethiopia

Jones et al. 2011 Ethiopia

Abere et al. 2013 Ethiopia

Puebla et al. 2015 Cuba

Alvarado-Esquivel et al. 2015 Mexico

Hadi and Faraj. 2016 Iraq

Strkolcova ct al. 2017 Slovakia

Paradics ct al. 2017 Italy

Sauda et al. 2018 Italy

Iatta et al. 2019 Italy

latta et al. 2019 ltaly

Dashchenko et al. 2020 Ukraine

Random effects model

Heterogeneity: $I^{2}=97.9031 \%, \tau^{2}=0.0580, p<.001$

Random effects model

Heterogeneity: $I^{2}=97.9974 \%, \tau^{2}=0.0540, p<.001$

Test for subgroup differences: $\chi_{1}^{2}=5.2672, \mathrm{df}=1(p=.02)$

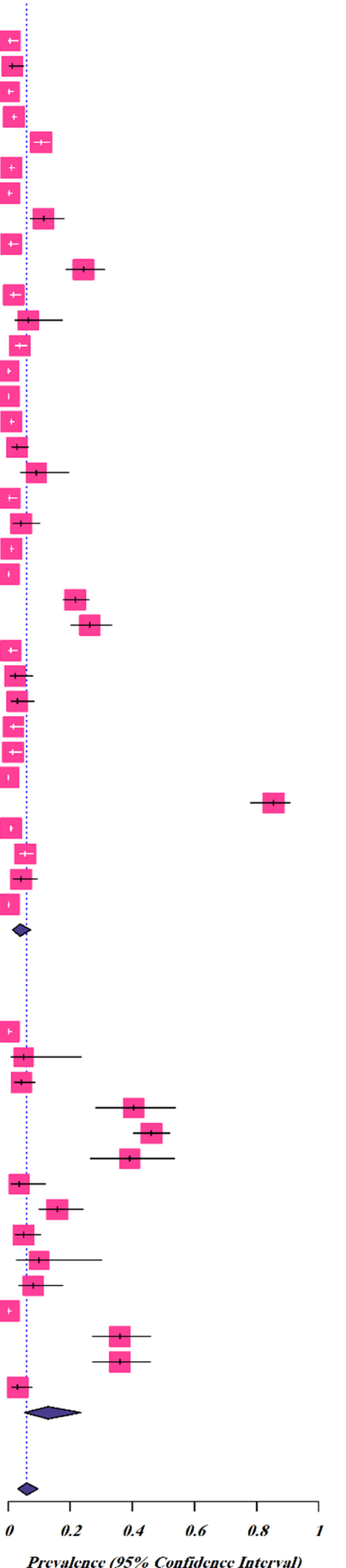

$0.0059 \quad[0.0010 ; 0.0326]$

$0.0140 \quad[0.0038 ; 0.0496]$

$0.0031 \quad[0.0006 ; 0.0174]$

$0.0186 \quad[0.0129 ; 0.0268]$

$0.1065 \quad[0.0835 ; 0.1349]$

$0.0104 \quad[0.0053 ; 0.0203]$

$0.0033 \quad[0.0009 ; 0.0118]$

$0.1145 \quad[0.0706 ; 0.1803\rfloor$

$0.0093 \quad[0.0026 ; 0.0333]$

$0.2431 \quad[0.1864 ; 0.3105]$

$0.0178 \quad[0.0076 ; 0.0410]$

$0.0652 \quad[0.0224 ; 0.1750]$

$0.0379 \quad[0.0231 ; 0.0615]$

$0.0012 \quad[0.0002 ; 0.0070]$

$0.0020 \quad[0.0012 ; 0.0034]$

$0.0109 \quad[0.0062 ; 0.0189]$

$0.0287 \quad[0.0123 ; 0.0655]$

$0.0909 \quad[0.0395 ; 0.1958]$

$0.0053 \quad[0.0009 ; 0.0294]$

$0.0417 \quad[0.0163 ; 0.1023]$

$0.0111 \quad[0.0072 ; 0.0172]$

$0.0025 \quad[0.0012 ; 0.0055\rfloor$

$0.2161 \quad[0.1779 ; 0.2600]$

$0.2632 \quad[0.2029 ; 0.3338 \mid$

$0.0084 \quad[0.0023 ; 0.0300]$

$0.0227 \quad[0.0063 ; 0.0791]$

$0.0300 \quad[0.0103 ; 0.0845]$

$0.0175 \quad[0.0060 ; 0.0503]$

$0.0152 \quad[0.0052 ; 0.0438]$

$0.0010 \quad[0.0003 ; 0.0028]$

$0.8547 \quad[0.7796 ; 0.9073]$

$0.0091 \quad[0.0056 ; 0.0148]$

$0.0546 \quad[0.0367 ; 0.0806]$

$0.0417 \quad[0.0179 ; 0.0938]$

$0.0020 \quad[0.0012 ; 0.0033]$

$0.0389[0.0154 ; 0.0725]$

$0.0022 \quad[0.0004 ; 0.0121]$

$0.0500 \quad[0.0089 ; 0.2361]$

$0.0429 \quad[0.0210 ; 0.0860]$

$0.4038 \quad[0.2816 ; 0.5393]$

$0.4610 \quad[0.4024 ; 0.5207]$

$0.3913 \quad[0.2639 ; 0.5354]$

$0.0357 \quad[0.0098 ; 0.1212]$

$0.1584 \quad[0.0999 ; 0.2419]$

$0.0500 \quad[0.0231 ; 0.1048]$

$0.1000 \quad[0.0279 ; 0.3010]$

$0.0806 \quad[0.0349 ; 0.1753]$

$0.0016 \quad[0.0003 ; 0.0088]$

$0.3600 \quad|0.2727 ; 0.4576|$

$0.3600 \quad[0.2727 ; 0.4576]$

$0.0308 \quad[0.0120 ; 0.0764]$

$0.1294[0.0521 ; 0.2346]$

$0.0603 \quad[0.0323 ; 0.0961]$

Fig. 3 Forest plots for random-effects meta-analysis of the global prevalence of Strongyloides stercoralis in owned and stray/shelter dogs 


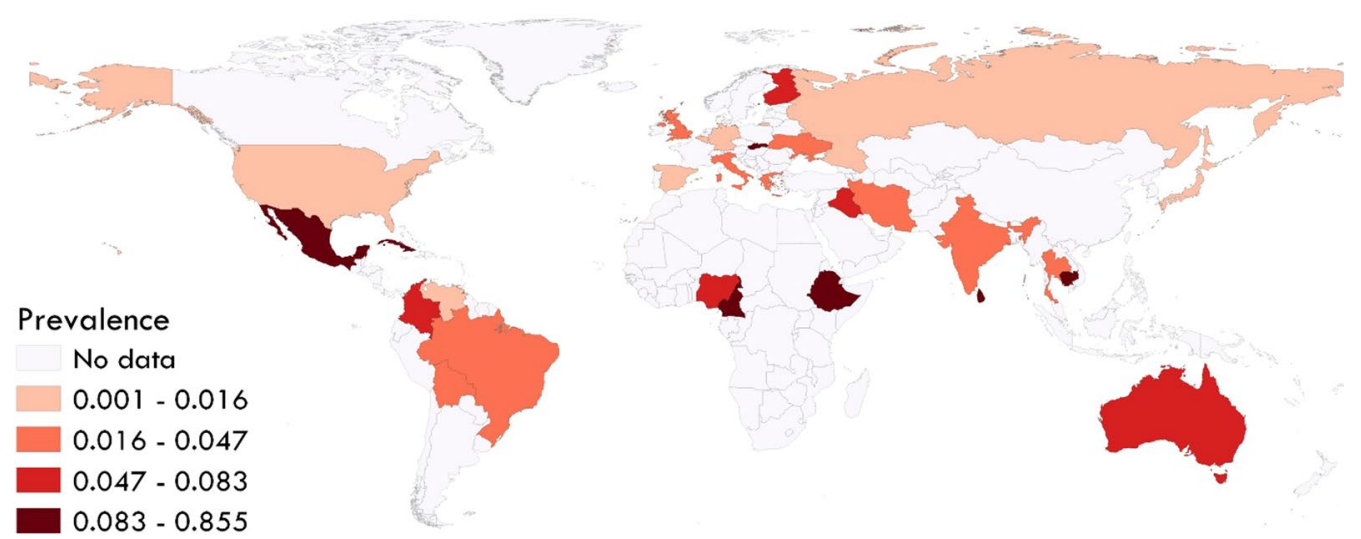

Fig. 4 Global prevalence of Strongyloides stercoralis in dogs based on included studies

access to veterinary care, specifically access to or affordability of anthelmintics, might be limited, contributing to the higher prevalence. Also, in low-income countries, the number of stray dogs can be high with the prevalence of S. stercoralis infection being greater than that in owned dogs, based on the data from the meta-analysis herein presented [41, 42].

Cambodia, where the highest prevalence was found in our analysis, serves as a potential example of the interaction of climate, income and free-roaming (individually or community owned but not contained) or stray dogs. Cambodia is a lower middle-income country with a tropical climate and limited accessibility to improved sanitation services as well as safe drinking water [43]. In the human population, S. stercoralis is a public health concern with prevalence being $>40 \%$, one of the higher levels in human populations that have been found [4446]. Lastly, many dogs in the country are free-roaming or stray [47]. These potential interactions support the need for a One Health approach to addressing Strongyloides infections in humans and dogs.

There is no gold standard method for detecting $S$. stercoralis in dogs (or people), and the current techniques have limited sensitivity due to the low burden of parasites and intermittent larval shedding [21, 48]. The most commonly used methods for the diagnosis of S. stercoralis infection in humans are direct smear and Kato-Katz, both of which have low sensitivity $[12,46]$. Sedimentation, the Baermann method and agar plate culture have higher sensitivity, but they are inconvenient, time-consuming and still underestimate infections $[12,49]$. While these latter methods were used in most of the studies included in the meta-analysis, technical details were inconsistent and their implementation varied. For example, with the Baermann the quantity of fecal material and the number of samples analyzed (e.g. one or three from consecutive days) were not standardized across studies, thus resulting in varied sensitivity of the method across studies. In some of the included articles, the focus was on general parasite prevalence with flotation and smears used for fecal analysis, standard screening methods for parasites in dogs but methods with low sensitivity for S. stercoralis, potentially resulting in an underestimation of prevalence. Interpretation of results from serological and molecular-based techniques, which were used in a few of the included articles, must be made with caution because of the possibility of false-positive and/or -negative results [21]. Given the high variation in how each diagnostic method was used in the studies, sensitivity ranges could not be assigned with confidence; hence, in the metaanalysis herein presented, prevalence was not adjusted based on the diagnostic method used with the resulting global prevalence likely underestimated.

In a clinical setting, S. stercoralis infections in dogs also are likely to be underestimated or overlooked because of the challenge of differentiating $S$. stercoralis larvae from other larvae that can occur in feces [i.e. Angiostrongylus vasorum, Crenosoma vulpis, Filaroides (Oslerus) osleri, Filaroides hirti and Filaroides milksi] [21]. Also, feces must be directly collected from the rectum or collected from the ground immediately after defecation to prevent fecal contamination with the larvae of free-living nematodes. 


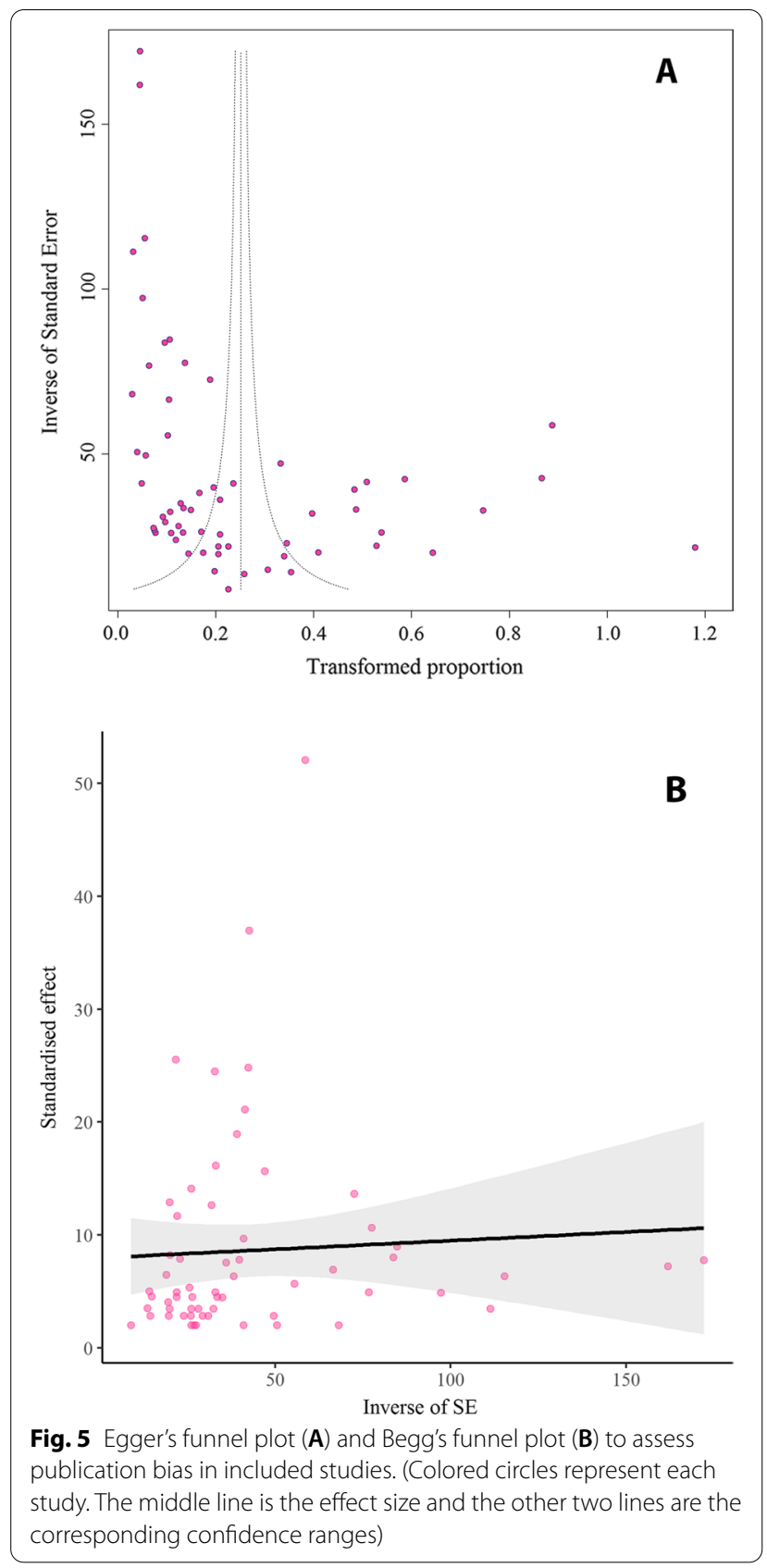

Most of the studies included in the meta-analysis were from tropical and subtropical regions, biasing the result towards higher prevalence in these regions. In other regions, within specific dog populations, prevalence might be higher than indicated in the meta-analysis with studies targeting S. stercoralis in susceptible dog populations (e.g. kennels, strays and shelter dogs) having prevalence similar to that seen in tropical and subtropical regions $[16,19,50]$. Hence, there is a need for more prevalence studies outside of tropical and subtropical regions to obtain a better understanding of the zoonotic risk.

\section{Conclusion}

The results of this systematic review and meta-analysis indicate the significant burden and current status of S. stercoralis infection in dogs in different parts of the world and highlight the need for studies in more geographical regions using methods with defined sensitivity. Paying attention to waste management systems, improving hygiene education and sanitary facilities in human populations as well as cleaning the environment of dog feces and establishing a preventive strategy for stray dogs could reduce the prevalence of the infection, especially in lower-income tropical and subtropical regions of the world. To decrease the burden of infective larvae in the environment contaminated with the feces of dogs, and in order to protect the canine and human population from the risk of infection, adequate deworming practices are essential. While few studies directly link infection of Strongyloides in humans to dogs, the shared genotypes and the similarity in where prevalence is higher support that the zoonotic potential of S. stercoralis infection is an important subject that should be reflected through raising awareness among dog owners and veterinarians. We recommend health authorities to organize efficient monitoring programs for protecting humans and dogs from the impact of the infection. 


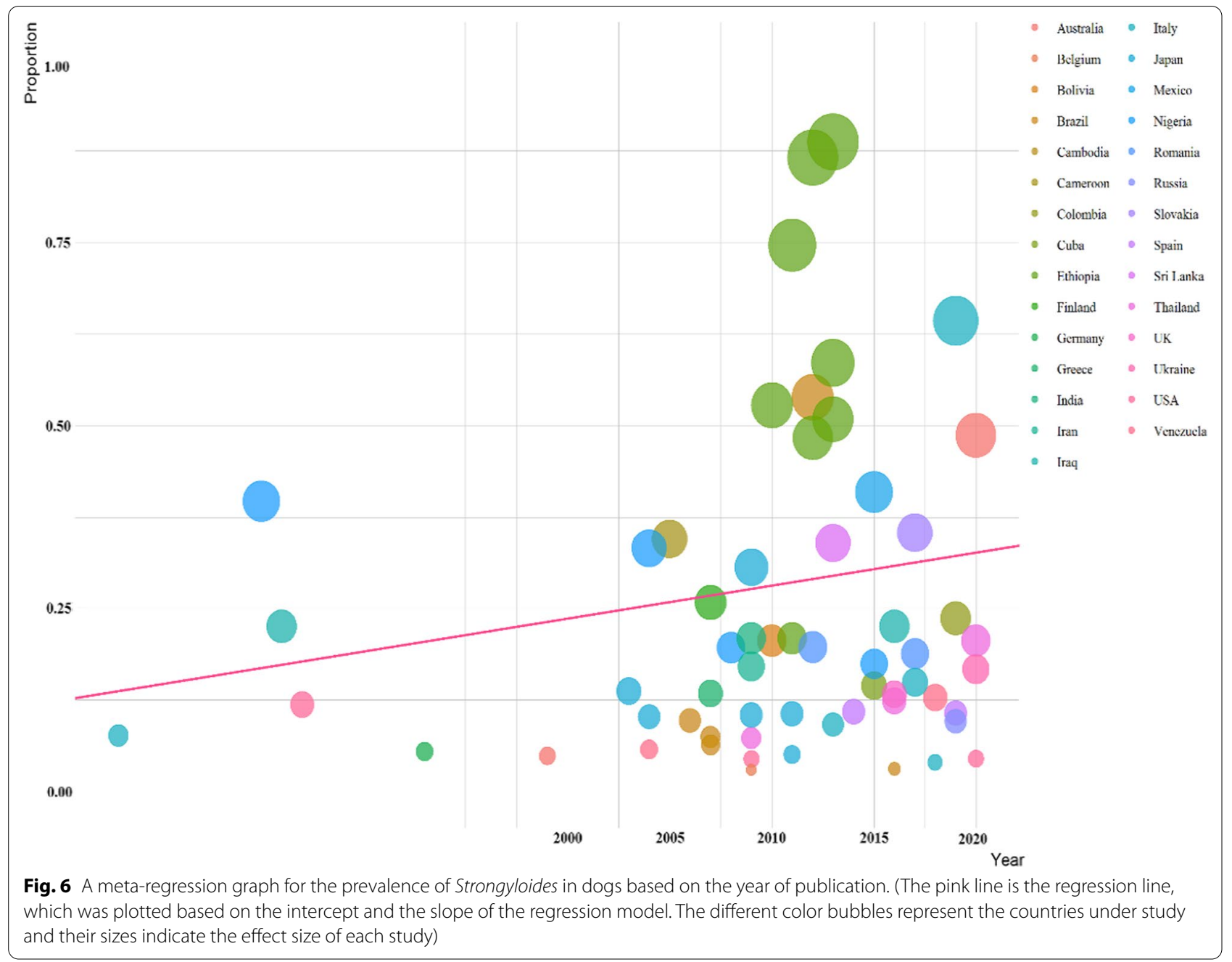

\section{Supplementary Information}

The online version contains supplementary material available at https://doi. org/10.1186/s13071-021-05135-0.

Additional file 1: References S1. List of articles used in the meta-analysis.

Additional file 2: Figure S1. Sub-group analysis of the prevalence of Strongyloides stercoralis in included studies based on country.

Additional file 3: Table S1. Sub-group analysis of the prevalence of Strongyloides stercoralis in included studies based on continent, income level, humidity, annual rainfall, average temperature, latitude and climate.

\section{Acknowledgements}

We sincerely thank personnel from the Metabolic Diseases Research Center, Research Institute for Prevention of Non-Communicable Diseases, Qazvin, Iran.

\section{Authors' contributions}

$M B, A V E$ and JKK designed the study. EH, EH and RM searched for primary publications, screened and appraised primary studies. AVE and MB extracted the data and, with JKK, wrote the study manuscript. MO, EH and SH contributed to data analysis and interpretation and edited the manuscript. All authors read the manuscript and participated in the preparation of the final version of the manuscript. All authors read and approved the final manuscript.

\section{Funding}

This work was supported by the Metabolic Diseases Research Center, Research Institute for Prevention of Non-Communicable Diseases, Qazvin, Iran (contract no. IR.QUMS.REC.1400.270).

\section{Availability of data and materials}

All data are included in the manuscript or as supplementary files.

\section{Declarations}

\section{Ethics approval and consent to participate}

Not applicable. No ethics approval or consent to participate were required for this work.

\section{Consent for publication}

Not applicable. No figures are included in this work that require consent for publication.

\section{Competing interests}

The authors have no competing interests.

\section{Author details}

${ }^{1}$ Medical Microbiology Research Center, Qazvin University of Medical Sciences, Qazvin, Iran. ${ }^{2}$ Metabolic Diseases Research Center, Research Institute 
for Prevention of Non-Communicable Diseases, Qazvin University of Medical Sciences, Qazvin, Iran. ${ }^{3}$ Department of Parasitology, Faculty of Veterinary Medicine, Rasht Branch, Islamic Azad University, Guilan, Iran. ${ }^{4}$ Department of Parasitology and Mycology, Qazvin University of Medical Sciences, Qazvin, Iran. ${ }^{5}$ Student Research Committee, Qazvin University of Medical Sciences, Qazvin, Iran. ${ }^{6}$ Ross University School of Veterinary Medicine, Basseterre, West Indies, St. Kitts and Nevis.

\section{Received: 20 August 2021 Accepted: 16 December 2021} Published online: 10 January 2022

\section{References}

1. Zibaei M, Nosrati MRC, Shadnoosh F, Houshmand E, Karami MF, Rafsanjani MK, et al. Insights into hookworm prevalence in Asia: a systematic review and meta-analysis. Trans R Soc Trop Med Hyg. 2020;114(3):141-54.

2. Omidinia N, Zibaei M, Hosseini H, Pourrostami K, Eslahi AV, Badri M. Human hydatidosis in Alborz province: a 5-year retrospective epidemiological analysis of hospitalized cases. Ann Parasitol. 2020;66(4):587-92.

3. Cleaveland S, Laurenson MK, Taylor LH. Diseases of humans and their domestic mammals: pathogen characteristics, host range and the risk of emergence. Philos Trans R Soc B Biol Sci. 2001;356:991-9.

4. Eslahi AV, Mowlavi G, Houshmand E, Pirestani M, Majidiani H, Nahavandi $\mathrm{KH}$, et al. Occurrence of Dioctophyme renale (Goeze, 1782) in road-killed canids of Iran and its public health implication. Vet Parasitol Reg Stud Rep. 2021;24:100568.

5. Eslahi AV, Badri M, Nahavandi KH, Houshmand E, Dalvand S, Riahi SM, et al. Prevalence of strongyloidiasis in the general population of the world: a systematic review and meta-analysis. Pathog Glob Health. 2021;115:7-20.

6. Ko PP, Suzuki K, Canales-Ramos M, Htwe MPPTH, Htike WW, Yoshida A, et al. Phylogenetic relationships of Strongyloides species in carnivore hosts. Parasitol Int. 2020;78:102151.

7. Barroso M, Salvador F, Sánchez-Montalvá A, Bosch-Nicolau P, Molina I. Strongyloides stercoralis infection: a systematic review of endemic cases in Spain. PLoS Negl Trop Dis. 2019;13(3):e0007230.

8. Viney M. Strongyloides. Parasitology. 2017;144(3):259-62.

9. Nutman T. Human infection with Strongyloides stercoralis and other related Strongyloides species. Parasitology. 2017;144(3):263-73.

10. Buonfrate D, Requena-Mendez A, Angheben A, Cinquini M, Cruciani $M$, Fittipaldo A, et al. Accuracy of molecular biology techniques for the diagnosis of Strongyloides stercoralis infection - a systematic review and meta-analysis. PLoS Negl Trop Dis. 2018;12(2):e0006229.

11. Levenhagen MA, Costa-Cruz JM. Update on immunologic and molecular diagnosis of human strongyloidiasis. Acta Trop. 2014;135:33-43.

12. Schär F, Trostdorf U, Giardina F, Khieu V, Muth S, Marti H, et al. Strongyloides stercoralis: global distribution and risk factors. PLoS Negl Trop Dis. 2013;7(7):e2288.

13. Barratt JLN, Lane M, Talundzic E, Richins T, Robertson G, Formenti F, et al. A global genotyping survey of Strongyloides stercoralis and Strongyloides fuelleborni using deep amplicon sequencing. PLoS NegI Trop Dis. 2019;13(9):e0007609.

14. Umur \$̧, Meral Y, Bölükbaş CS, Gürler AT, Acici M. First clinical Strongyloides stercoralis case in a dog in Turkey. Turk J Vet Anim Sci. 2017;41 (2):312-5.

15. Goncalves ALR, Machado GA, Goncalves-Pires MRF, Ferreira-Junior A, Silva DAO, Costa-Cruz JM. Evaluation of strongyloidiasis in kennel dogs and keepers by parasitological and serological assays. Vet Parasitol. 2007; 147(1-2):132-9.

16. Dillard KJ, Saari SAM, Anttila M. Strongyloides stercoralis infection in a Finnish kennel. Acta Vet Scand. 2007:49(1):1-6.

17. Júnior AF, Gonçalves-Pires MRF, Silva DAO, Goncalves ALR, Costa-Cruz JM. Parasitological and serological diagnosis of Strongyloides stercoralis in domesticated dogs from southeastern Brazil. Vet Parasitol. 2006;136(2):137-45

18. Eydal M, Skírnisson K. Strongyloides stercoralis found in imported dogs, household dogs and kennel dogs in Iceland. Icel Agric Sci. 2016;29:39-51.

19. Dashchenko S, Soroka N, Semenko O. Distribution of Strongyloides stercoralis among dogs of different housing groups in Kyiv and Kyiv region, clinical manifestations and diagnostic methods. EUREKA Health Sci. 2020;5:99-107.

20. Basso W, Grandt L-M, Magnenat A-L, Gottstein B, Campos M. Strongyloides stercoralis infection in imported and local dogs in Switzerland: from clinics to molecular genetics. Parasitol Res. 2019;118(1):255-66.

21. Paradies P, larussi F, Sasanelli M, Capogna A, Lia RP, Zucca D, et al. Occurrence of strongyloidiasis in privately owned and sheltered dogs: clinical presentation and treatment outcome. Parasit Vectors. 2017;10(1):1-9.

22. Thamsborg SM, Ketzis J, Horii Y, Matthews JB. Strongyloides spp. infections of veterinary importance. Parasitology. 2017;144(3):274-84.

23. Faust EC, Kagy ES. Experimental studies on human and primate species of Strongyloides. I. The variability and instability of types. Am J Trop Med. 1933;13:47-65.

24. Lok JB, Community TCeR. Strongyloides stercoralis: a model for translational research on parasitic nematode biology. WormBook. 2007. https:// doi.org/10.1895/wormbook.1.134.1.

25. Buonfrate D, Bisanzio D, Giorli G, Odermatt P, Fürst T, Greenaway C, et al. The global prevalence of Strongyloides stercoralis infection. Pathogens. 2020;9(6):468.

26. Wells G, Shea B, O'Connell D, Peterson J, Welch V, Losos M, et al. The Newcastle-Ottawa Scale (NOS) for assessing the quality if nonrandomized studies in meta-analyses. www.ohri.ca/programs/clinical_epidemiology/ oxford.asp. Accessed 3 July 2021.

27. Badri M, Eslahi AV, Olfatifar M, Dalvand S, Houshmand E, et al. Keys to unlock the enigma of ocular toxocariasis: a systematic review and metaanalysis. Ocul Immunol Inflamm. 2021;12:1-2.

28. Eslahi AV, Olfatifar M, Abdoli A, Houshmand E, Johkool MG, Zarabadipour M, et al. The neglected role of Trichomonas tenax in oral diseases: a systematic review and meta-analysis. Acta Parasitol. 2021;66:715-32.

29. Mirzadeh M, Olfatifar M, Eslahi AV, Abdoli A, Houshmand E, Majidiani $\mathrm{H}$, et al. Global prevalence of Trichomonas vaginalis among female sex workers: a systematic review and meta-analysis. Parasitol Res. 2021;120(7):2311-22.

30. Kanat-Maymon Y, Antebi A, Zilcha-Mano S. Basic psychological need fulfillment in human-pet relationships and well-being. Pers Individ Dif. 2016;92:69-73.

31. Grajfoner D, Harte E, Potter LM, McGuigan N. The effect of dog-assisted intervention on student well-being, mood, and anxiety. Int J Environ Res Public Health. 2017;14(5):483.

32. Ritossa L, Viozzi G, Flores V. The state of knowledge on intestinal helminths in free-roaming dogs in southern South America. Canine Genet Health Med. 2021. https://doi.org/10.5772/intechopen.96125.

33. Ghasemzadeh I, Namazi SH. Review of bacterial and viral zoonotic infections transmitted by dogs. J Med Life. 2015;8(Spec Iss 4):1.

34. Szabová E, Juriš P, Miterpáková M, Antolová D, Papajová I, Šefčíková H. Prevalence of important zoonotic parasites in dog populations from the Slovak Republic. Helminthologia. 2007;44(4):170-6.

35. Jaleta TG, Zhou S, Bemm FM, Schär F, Khieu V, Muth S, et al. Different but overlapping populations of Strongyloides stercoralis in dogs and humans-dogs as a possible source for zoonotic strongyloidiasis. PLOS Negl Trop Dis. 2017;11(8):e0005752.

36. Viney ME, Lok JB. The biology of Strongyloides spp. WormBook. 2015;16:1-17

37. Paula FM, Costa-Cruz JM. Epidemiological aspects of strongyloidiasis in Brazil. Parasitology. 2011;138(11):1331-40.

38. Sartorius B, Cano J, Simpson H, Tusting LS, Marczak LB, Miller-Petrie MK, et al. Prevalence and intensity of soil-transmitted helminth infections of children in sub-Saharan Africa, 2000-18: a geospatial analysis. Lancet Glob Health. 2021;9(1):e52-60.

39. De Silva NR, Brooker S, Hotez PJ, Montresor A, Engels D, Savioli L. Soiltransmitted helminth infections: updating the global picture. Trends Parasitol. 2003;19(12):547-51.

40. Beknazarova M, Whiley H, Ross K. Strongyloidiasis: a disease of socioeconomic disadvantage. Int J Environ Res Public Health. 2016;13(5):517.

41. Abulude OA. Prevalence of intestinal helminth infections of stray dogs of public health significance in Lagos metropolis, Nigeria. Int Ann Sci. 2019;9(1):24-32.

42. Otranto D, Dantas-Torres F, Mihalca AD, Traub RJ, Lappin M, Baneth G. Zoonotic parasites of sheltered and stray dogs in the era of the global economic and political crisis. Trends Parasitol. 2017;33(10):813-25. 
43. Liao C-W, Chiu K-C, Chiang I-C, Cheng P-C, Chuang T-W, Kuo J-H, et al. Prevalence and risk factors for intestinal parasitic infection in schoolchildren in Battambang, Cambodia. Am J Trop Med Hyg. 2017;96(3):583.

44. Forrer A, Khieu V, Schindler C, Schär F, Marti H, Char MC, et al. Ivermectin treatment and sanitation effectively reduce Strongyloides stercoralis infection risk in rural communities in Cambodia. PLoS Negl Trop Dis. 2016;10(8):e0004909.

45. Khieu V, Schär F, Forrer A, Hattendorf J, Marti H, Duong S, et al. High prevalence and spatial distribution of Strongyloides stercoralis in rural Cambodia. PLoS Negl Trop Dis. 2014;8(6):e2854.

46. Eslahi AV, Olfatifar M, Karim MR, AbuOdeh R, Modirian E, Houshmand E, et al. Global incidence of helminthic contamination of vegetables, cucurbits and fruits: a systematic review and meta-analysis. Food Control. 2021. https://doi.org/10.1016/j.foodcont.

47. Chevalier V, Davun H, Sorn S, Ly P, Pov V, Ly S. Large scale dog population demography, dog management and bite risk factors analysis: a crucial step towards rabies control in Cambodia. PLoS ONE. 2021;16(7):e0254192.

48. latta R, Buonfrate D, Paradies P, Cavalera MA, Capogna A, larussi F, et al. Occurrence, diagnosis and follow-up of canine strongyloidiosis in naturally infected shelter dogs. Parasitology. 2019;146(2):246-52.

49. Vafae Eslahi A, Olfatifar M, Houshmand E, Ghanbari Johkoold M, Zibaei M, Foroutan M, Hosseinie H, Badri M, et al. Prevalence of Strongyloides stercoralis in the immunocompetent and immunocompromised individuals in Iran: a systematic review and meta-analysis. Trans R Soc Trop Med Hyg. 2021. https://doi.org/10.1093/trstmh/trab104.

50. Gates MC, Nolan TJ. Endoparasite prevalence and recurrence across different age groups of dogs and cats. Vet Parasitol. 2009;166(1-2):153-8.

\section{Publisher's Note}

Springer Nature remains neutral with regard to jurisdictional claims in published maps and institutional affiliations.

- fast, convenient online submission

- thorough peer review by experienced researchers in your field

- rapid publication on acceptance

- support for research data, including large and complex data types

- gold Open Access which fosters wider collaboration and increased citations

- maximum visibility for your research: over 100M website views per year

At BMC, research is always in progress.

Learn more biomedcentral.com/submissions 